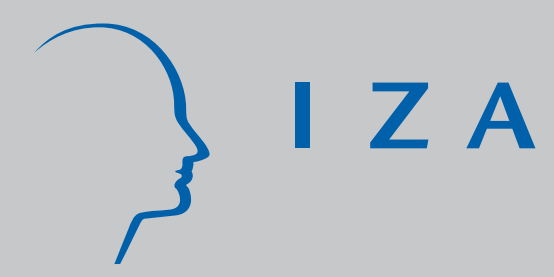

IZADP No. 1697

Do Social Preferences Increase Productivity?

Field Experimental Evidence from

Fishermen in Toyama Bay

J effrey Carpenter

Erika Seki

J uly 2005 


\title{
Do Social Preferences Increase Productivity? Field Experimental Evidence from Fishermen in Toyama Bay
}

\author{
Jeffrey Carpenter \\ Middlebury College and IZA Bonn \\ Erika Seki \\ University of Aberdeen
}

Discussion Paper No. 1697

July 2005

IZA

P.O. Box 7240

53072 Bonn

Germany

Phone: $+49-228-3894-0$

Fax: +49-228-3894-180

Email: iza@iza.org

Any opinions expressed here are those of the author(s) and not those of the institute. Research disseminated by IZA may include views on policy, but the institute itself takes no institutional policy positions.

The Institute for the Study of Labor (IZA) in Bonn is a local and virtual international research center and a place of communication between science, politics and business. IZA is an independent nonprofit company supported by Deutsche Post World Net. The center is associated with the University of Bonn and offers a stimulating research environment through its research networks, research support, and visitors and doctoral programs. IZA engages in (i) original and internationally competitive research in all fields of labor economics, (ii) development of policy concepts, and (iii) dissemination of research results and concepts to the interested public.

IZA Discussion Papers often represent preliminary work and are circulated to encourage discussion. Citation of such a paper should account for its provisional character. A revised version may be available directly from the author. 


\section{ABSTRACT}

\section{Do Social Preferences Increase Productivity? Field Experimental Evidence from Fishermen in Toyama Bay*}

We provide a reason for the wider economics profession to take social preferences, a concern for the outcomes achieved by other reference agents, seriously. Although we show that student measures of social preference elicited in an experiment have little external validity when compared to measures obtained from a field experiment with a population of participants who face a social dilemma in their daily lives (i.e., team production), we do find strong links between the social preferences of our field participants and their productivity at work. We also find that the stock of social preferences evolves endogeously with respect to how widely team production is utilized.

JEL Classification: C93, D21, D24, H41, J24, M52, M54, Z13

Keywords: field experiment, social preference, income pooling, productivity

Corresponding author:

Jeffrey P. Carpenter

Department of Economics

Middlebury College

Middlebury, VT 05753

USA

Email: jpc@middlebury.edu

\footnotetext{
* We thank Kiyoshi Yokoo for research assistance and the National Science Foundation (CAREER 0092953) for financial support. In addition, we recognize the helpful comments of Abigail Barr, Juan Camilo Cardenas, Hugo Nopo, Peter Matthews, Will Pyle, and Pieter Serneels.
} 


\section{Introduction}

Laboratory experiments in economics have provided overwhelming evidence that many student participants exhibit social preferences: they care about the outcomes achieved by reference agents in addition to their own outcomes. These preferences include conditional cooperation (Fischbacher et al., 2001) and the propensity to punish free riders (Fehr and Gaechter, 2000b) and have been shown to transform various social dilemmas like team production into coordination problems in which participants often achieve Pareto efficient outcomes. However, the laboratory data suffer two shortcomings. One problem, that has resulted in many recent field experiments, is that it is hard to know how well student responses in the lab generalize to other important populations - the external validity problem. A second related problem is that experimentalists have provided little reason for other economists to care about social preferences. That is, showing that non-students exhibit social preferences is not the same as showing that social preferences have an impact on economic outcomes. ${ }^{1}$ Until experiments establish direct links in relevant populations between experimental measures of social preference and economic outcomes, these ideas will continue to have limited impact.

While there are a growing number of field experiments to examine how well the results of student lab experiments generalize to different populations, there are very few experiments that (convincingly) link behavioral measures from experiments to economic outcomes, and, to our knowledge, none that accomplish both goals. Harrison and List (2004) review the recent literature on economic field experiments but are mostly silent on the topic of social preferences. In other specific experiments, Henrich (2000) and Cardenas et al. (2000) pioneered conducting social preference experiments in the field. For example, Henrich (2000) showed that one would have to travel to Amazonia to find participants who look remotely like the self-interested agent that much of traditional economic theory is built around and Cardenas et al. (2000) demonstrated how institutions can crowd out social preferences for cooperation among rural farmers and craftspeople in Colombia. More recently, Henrich et al. (2001) extend the earlier experiments to fifteen different cultures, Carpenter et al. (2005) show important differences exist between blue collar workers and students in distribution experiments, and List (2004) finds similar differences using the voluntary contribution mechanism.

With respect to the link between social preferences and economic outcomes, Karlan (2002) has shown that those participants who were more trustworthy in an experiment were more likely to repay their loans one year later. Carter and Castillo (2002) conduct trust and distribution games in South Africa and find that their experimental measures of altruism and trust are positively associated with household expenditures. Cardenas and Carpenter (2005) conduct a similar analysis of the links between experimental measures of cooperation from public goods games played by urban slum dwellers in Vietnam and Thailand

\footnotetext{
${ }^{1}$ Fehr and Fischbacher (2002), however, provide some convincing reasons why the profession should take lab experiments with students seriously.
} 
and household expenditures and find that more cooperative players were better off in Thailand but not Vietnam. Lastly, and more relevant for our purposes, Barr and Serneels (2004) find that individual experimental measures of trustworthiness are positively associated with the wages of Ghanaian manufacturing workers, however any link to productivity is only inferential.

The experiment that we report on in this paper is unique because it addresses both shortcomings simultaneously and therefore is the first research to directly link standard social preferences that have been identified in hundreds of lab experiments with topics that are at the core of economic theory. To accomplish this goal, we conducted a series of experiments in Toyama Prefecture, Japan. Our participants belonged to one of two groups; they were either local college students or fishermen who catch shrimp in the bay. The comparison of these two groups provides us with a link to the social preference literature based on traditional lab experiments and allows us to explore the external validity of student social preferences. We also collected productivity data from our fishermen that allow us to examine whether social preferences, measured with our experiment, have an impact on fishing productivity.

The fishermen we study are particularly well suited for this sort of research because they engage in team production and they have organized themselves into two distinct groups based on whether or not they pool their catch at the end of the day. The first factor implies that these fishermen face social dilemma incentives each day at work and therefore may rely on social preferences to attenuate shirking. In other words, there is some reason to hypothesize that social preferences will affect the productivity of our fishermen. The second factor (that some fishermen pool their catch) implies that the incentives for the two groups of fishermen are different. Although each boat, pooler or not, must contend with shirking among the crew members, this problem is compounded for the poolers because each pooling boat also confronts the incentive to free ride on the fishing effort of the other boats. This suggests that social preferences might develop to a different degree aboard pooling boats and might have a differential effect on their productivity.

We discuss the details of fishing in Toyama bay in the next section. In section 3 we describe our experiment. In section 4 we summarize the demographic characteristics of our participants and discuss the differences in overall experimental behavior between students, poolers and nonpoolers. In section 5 we present our methodology for constructing measures of social preference from the experimental data. In section 6 we ask whether the distributions of social preference differ based on whether our fishermen pool or not and whether these differences can be attributed to a treatment effect of pooling on fishermen preferences. In section 7 we present our main results that link social preferences to fishing productivity and we offer a few concluding remarks in section 8. 


\section{Shiroebi Fishing in Toyama Prefecture}

Toyama prefecture, located on the west coast of the Honsyu main island of Japan, has a semi-urban local economy dominated by traditional fishing industries that prosper because of their proximity to the large urban markets of Tokyo and Osaka. Toyama Bay, opening into the Sea of Japan, is known for a large variety of highly valued fish species because of the complex structure of the sea bottom and a mixture of warm and cold currents flowing into the bay. There are 16 small fishing communities situated along the coast including our base, Shinminato.

Coastal fisheries in Japan are managed by Fishery Cooperative Associations (FCAs). Local FCAs do not only implement and enforce national and prefectural legislation, they also devise complementary regulations that are designed specifically to deal with evolving local conditions. Within each FCA, fishermen are grouped according to the species they target and the fishing technologies that they use. The fishermen that we study belong to the $e b i$ self-managing group that specializes in shrimp fishing. Shiroebi (Japanese glass shrimp) fishing takes place between April and October - the season authorized by the FCAs. For the rest of the year, the fishermen are engaged in trawl fishing of crabs and cold-water shrimp (Akaebi). Before the late 1980s, shiroebi fishing was regarded as an auxiliary activity for the summer months by some of the fishermen.

The fishermen who catch shiroebi in the bay near Shinminato are organized into two groups. The members of the two groups live in Shinminato, belong to the same local FCA, use the same harbor facilities, and operate in the same fishery. However, there are two major differences between these groups: whether or not they pool their catch and their experience in shiroebi fishing. One group, consisting of seven boats and 30 fishermen ( 7 skippers, 21 crew members, and 2 onshore helpers) whom we call poolers, have been fishing shiroebi for generations and have shared both income and operating expenses since the 1960s. ${ }^{2}$ The second group, which we call the nonpoolers, consists of 5 fishing boats and 30 fishermen ( 5 skippers and 25 crewmen). Members of this second group do not pool income or expenses and began shiroebi fishing only in 1992.

Since the motorization of fishing boats in the early 1930's, competition among fishing units for access to the shiroebi stock has become a chronic problem. Many anecdotal stories testify to heavy congestion in the most favorable fishing spots, over-extraction, and frequent interpersonal conflicts. The early 1960's witnessed the implementation of advanced fishing equipment such as the echo-sounder (a.k.a., sonar), sophisticated navigation systems, and synthetic nets. The echo-sounder increased precision in detecting stock concentrations. Satellite-driven navigation systems have allowed skippers to remember and travel quickly to good fishing areas, as well as to operate under bad conditions. Synthetic materials allowed the fishermen to use larger nets and increase their average catch per haul.

\footnotetext{
${ }^{2}$ More specifically, poolers share both income and operating expenses, but in terms of capital investments, only the costs of nets are borne by the whole group; boats and other equipment are individually financed.
} 
At the same time however, the adoption of sophisticated and highly effective technical equipment by an ever larger number of competing fishermen led to more conflicts over the limited fishing spots. As a result, the catch per unit of effort (i.e., the kilos caught per trip into the bay) started to decline and the amount of net damage increased because the new synthetic materials emboldened skippers to take more risks. If these problems were not enough, during the same period fishermen in Toyama Bay increasingly suffered from active collusion among the fish merchants and middlemen. Ample opportunities existed for the merchants to collude and force fishermen to sell their catch at lower prices. To protest (and to increase their market power) the fishermen boycotted the traders' cartels in 1961 and pressured the FCA to organize fish auctions with the hope of increasing merchant competition. This demand was met and resulted in the demise of the merchant cartel.

It was immediately after the success of this collective protest, that the shiroebi fishermen in Shinminato organized and adopted the pooling system that has been maintained to this day. For thirty years the poolers were the only shiroebi fishers operating in Shinminato. This arrangement ended with the entrance of the nonpoolers in 1992. The nonpoolers, used to continue to harvest Akaebi and Toyama ebi between June and September. Since the mid 1980's, however, the economic importance of these fisheries has declined due to depressed unit prices for Akaebi and to the extinction of other species. Concurrently, shiroebi fishing benefited from the growing demand for fresh products due mostly to advancements in refrigerated transportation to the urban consumption centers. These events prompted the nonpoolers to request permission from the local FCA to begin fishing shiroebi. After six long years of negotiation with the poolers under the auspices of the local FCA, an arrangement was settled on under which the nonpolers were allowed to fish shiroebi on Tuesdays, Thursdays, and Saturdays between June and September while the poolers limited themselves to fishing on Mondays, Wednesdays, and Fridays.

Daily fishing operations start before sunrise (between 3:00 and 4:00 am) when all the boats belonging to the group set off. On any given fishing day, each boat will make a maximum of four or five hauls. Each haul takes about two hours to complete. A single haul consists of the following tasks. Once they have left the harbor, each skipper starts to search for promising fishing spots using his echo-sounder and radar. The skipper may record his position with the help of a global positioning satellite system. Among the poolers, one of the senior skippers is experienced in identifying the promising areas. This individual advantage generates externalities for all the pooling boats because the pooling skippers exchange information by radio. There is no such cooperation among the nonpooling skippers who actually take measures to conceal information about favorable fishing spots.

The fishing technique is called purse seining: each boat sets its net by encircling an area of concentration and then allows the net to drift. The shrimp gather at the end of the net after dragging it for some time at the appropriate speed. Success requires taking account of the current and seabed. Because the seabed in Toyama Bay is highly variable and the large net will be spread over 
a considerable distance (a rectangle approximately 100 by 200 meters), it becomes crucial to monitor the position of the net to target the stock accurately while avoiding ensnaring the net and damaging it. Damaged nets are very costly because they are complicated to repair and the opportunity cost of net repair time is very high.

To improve the precision of targeting stock concentrations and avoid damaging nets, the poolers occasionally adopt pair-fishing. The skipper of one boat sets his net while another skipper positions his boat above the net. The skipper of the second boat can then closely monitor the location of the fish and the position of the net with his echo-sounder. Likewise, he can give the skipper on the net-laying boat instructions about the desirable speed and duration of drift.

After trawling for some time, the crew gradually reels in the net and removes the shrimp. The shrimp are stored in containers of iced water for the journey back to port. This journey is competed as quickly as possible to avoid deterioration of the catch. Once in port, the haul is weighed and transferred directly to the traders (i.e., the auctioneers). All landings must be completed by 1pm which is the time of the final auction for the day.

As hinted at above, the poolers face different incentives. On one hand, pooling provides the same effort problems as team production: each crew has the incentive to free ride off the efforts of the other crews. On the other hand, the poolers also have the incentive to share information and fish cooperatively. Indeed, poolers exhibit more cooperation in terms of work coordination, effort regulation, and the sharing of information and expertise. In addition to pairfishing, work coordination includes concerted searches for stock, coordination of access to fishing spots, and collective net maintenance (including the search for lost nets). In addition, collective effort regulation prevents individual fishermen from unprofitable overproduction and sharing information and expertise enables all the poolers to take advantage of individual research on innovative fishing techniques.

The pooler's coordination seems to yield productivity gains. In terms of their fishing effectiveness, poolers tend to catch more than nonpoolers. ${ }^{3}$ This difference was first recognized in Platteau and Seki (1998) and was confirmed almost five years later during our stay in Shinminato, because we collected data on individual hauls from 115 trips into the bay. Our estimation of the fishing production function discussed in more detail in section 7 suggests that poolers typically catch 0.4 standard deviations more per trip into the bay than nonpoolers and this difference is significant at the $1 \%$ level.

While we suspect that the teamwork that poolers engage in at the boat level accounts for much of this difference in productivity, we also wondered whether

\footnotetext{
${ }^{3}$ The poolers' better performance is not limited to their productivity. During the months in which they fish for shiroebi by themselves (April and May), the poolers are effective at acting collectively as a monopolist and have become skilled at price discrimination. Therefore, the poolers also have higher average monthly revenues. This aspect of the difference between poolers and nonpoolers is analyzed in depth in Seki (2004). Our current analysis uses data from the period in which both groups are active. During this period the two groups compete for larger hauls.
} 
social preferences could explain the difference. Indeed, our extensive interviews with skippers and crewmen provide the foundation for this hypothesis. In these interviews, the fishermen spoke freely about their professional lives and their interactions with the other fishermen. They expressed opinions not only regarding technical and environmental aspects of their work but also regarding the social and economic aspects of their experiences with pooling. As a matter of fact, a number of statements made by the fishermen indicate the emergence of inter-personal comparisons of productivity and social preferences for cooperative behavior among the poolers. ${ }^{4}$ With these interviews in mind, and informed by Bowles (1998), we hypothesized that cooperative social preferences (e.g., the propensities to cooperate, punish free riding, and respond prosocially to punishment) might evolve to a greater extent on the pooling boats and that these preferences might contribute to higher productivity perhaps on their own or perhaps by laying the foundation for the cooperative activities mentioned above. To test this hypothesis, we conducted a field experiment to measure the social preferences of the fishermen.

\section{Our Field Experimental Design}

As we described in the previous section, our fishermen rely on teamwork to catch shrimp and therefore may face the problem of shirking. To increase the external validity of our results, and perhaps the saliency of the protocol for our participants, we choose an experiment that simulated the incentives that these participants face on their boats. This motivation lead us to use the experiment first discussed in Carpenter et al. (2004b) and Carpenter et al. (2004a) which combines a standard, hand-run, version of the familiar repeated voluntary contribution mechanism (VCM) with a modification in which participants can pay to show their disapproval of the contribution behavior of the other members of the group. Because theorists have shown that this sort of disapproval and the resulting shame can attenuate the incentive to shirk in teams (e.g., Kandel and Lazear, 1992), we decided that the "social disapproval" game best suited our purposes. The game allowed us to gather data on the willingness of team members to show their disapproval and their responsiveness to displayed disapproval, in addition to the contribution data that is typically collected in the VCM.

In our version of the social disapproval experiment, participants were divided into four person groups that maintained the same membership for ten rounds. Each group member was endowed with ten 50 yen coins and was asked to privately contribute as many coins as (s)he wanted to a public good (i.e.,

\footnotetext{
${ }^{4}$ Here are a few of the statements purporting the emergence of cooperative preferences: "Because we pool landings, I always feel under pressure to having good catches"; "It is out of the question to become lazy because we share income. On the contrary, income pooling prompts me to work more thoughtfully"; "Fishers with higher catches will be indifferent about (i.e., will not disapprove of) those with lower catches. On the contrary, the former will help the latter to improve by teaching them where the better spots to fish are."; "We (poolers) have team sprit to perform better as a whole."
} 
the experiment was single blind). At the time we conducted the experiments (August and September, 2003) the per-round endowment was equal to $\$ 4.30$ which was a substantial sum for our participants. ${ }^{5}$ Once all the participants had made their contribution decisions, the total contribution was calculated for each group, each group total was doubled, and then the proceeds were distributed equally to the group members. This implies that the marginal per capita return from the public good was 0.5 : each coin contributed was doubled by the experimenters and then divided four ways. Because the marginal return to contributing a coin is lower than the payoff to keeping the coin, and this calculus does not depend on the level of group contributions, the dominant strategy is to contribute nothing even though the team members double their money when they all contribute fully.

The first five rounds of the experiment replicated the VCM and then in the last five rounds we instituted the social disapproval protocol. When the first round began, the participants were unaware of how the protocol would change after round five. We choose this design feature so that behavior in the first five rounds would not be confounded by the anticipation of the rule change. The first five rounds followed a hand-run protocol that is now standard in the literature (see Ledyard, 1995 and the instructions for both segments that appear in Appendix A), but the social disapproval institution is an innovation that is important to discuss in some detail.

During the social disapproval rounds, the first half of the protocol proceeded exactly as in the standard VCM. Participants decided how much of their endowment to contribute and then were informed of the group total contribution (and shown the individual contributions in random order) and their payoff from the public good. After being shown the distribution of contributions and the group total contribution, participants were asked if they wanted to anonymously send a signal to the rest of the group. This signal, an unhappy face (which has a similar connotation in Japanese culture) cost ten yen and was displayed for everyone in the group to see at the beginning of the next round. Therefore, at the beginning of rounds 7 through 10 , each group member saw between zero and four unhappy faces that signaled the number of group members who were unhappy with the level of the public good or the distribution of individual contributions provided in the previous round.

While the social disapproval protocol has the flavor of the punishment experiments described in Fehr and Gaechter (2000a) and Carpenter (2004), for example, it is substantially different because punishment carries no material penalty (a la Masclet et al., 2003) and it is not directed at individuals (which would be hard to implement by hand in the field for more than one round). ${ }^{6}$

\footnotetext{
${ }^{5}$ In fact, the experiment typically lasted less than an hour and our participants earned $\$ 73.19$, on average.

${ }^{6}$ There is an important difference between our social disapproval protocol and the Masclet et al. (2003) experiment. In Masclet et al., it was free to send signals which implies that there are many equilibria in which punishment is a component. By imposing a cost, the social disapproval protocol assures that the only subgame perfect equilibrium of the underlying game is the same as in the VCM. Specifically, the cost associated with sending a signal motivates
} 
At the same time, the simplicity of the protocol is elegant because the stark incentives make it clear that there are no strategic reasons to send a signal and if people do send signals (and respond to them) then we have particularly compelling measures of social preference.

We conducted four sessions of the experiment with 53 participants. To provide a link to the standard experimental literature, 26 of these participants were students recruited from nearby universities. ${ }^{7}$ The two fishermen sessions took place in a large meeting room at the fishing coop and the two student sessions were conducted in a large conference room that we rented at a local hotel. The procedures were single blind anonymous and our participants made their decisions privately behind portable blinds that we constructed. ${ }^{8}$ At the beginning of each round, participants were given envelopes with ten 50 yen coins inside. We decided to use endowments of real money to make the choices as salient as possible. The first decision for each individual was to transfer as many of the coins as he wanted from the small endowment envelop to a large opaque envelop. When everyone was finished, the experimenters collected the small envelopes and computed the distribution of contributions and the group total contributions. On slips of paper, the experimenters wrote, in random order, the individual contributions to the public good, the group total contribution, and the individual payout from the public good. These slips were distributed to the participants as feedback. While the participants were making their decisions, the third experimenter entered the data into a laptop so that it would be fast and easy to calculate each participant's final payoff at the end of the experiment. ${ }^{9}$

At the end of round five the experiment was interrupted and the social disapproval protocol was explained. Participants made their contribution decisions and received feedback on the behavior of their group as before, but then they were given slips of paper on which they indicated whether they wanted to send a signal (at a cost of 10 yen) to the group or not. To display the signals, we stamped between zero and four unhappy faces on the front of the smaller endowment envelopes at the beginning of the next round. Therefore, at time $t$ each participant saw $n$ signals that indicated that $n$ members of the group were unhappy with how much the group contributed in period $t-1$.

participants to free ride off any disapproval done by the other group members and, as a result, no signals should be sent. Additionally, social disapproval imposes no monetary penalty which implies egoistic participants should ignore it. Combined these incentives suggest the dominant strategy is to free ride at both opportunities.

${ }^{7}$ Neither 53 nor 26 is evenly divisible by four. In three cases we did not want to turn away participants from our limited subject pools because we did not have enough people to form another group of four. Instead, we relied on the fact that participants could not know who the other members of their group were and formed groups with "shadow members." These randomly chosen shadow members contributed to their own group but their behavior was also counted in another group to get the total up to four persons.

${ }^{8}$ We owe a large debt of gratitude to our insightful research assistant, Kiyoshi Yokoo who not only helped us streamline our procedures, but was rock-solid during the experiments.

${ }^{9}$ The opportunity cost of fisherman time is extremely high and therefore we settled on procedures that made the experiment run as quickly as possible. 


\section{A Summary of our Participants and their Group- Level Behavior}

After the experiment was completed and while we calculated the final payoffs, the participants completed a demographic and attitudinal survey. The top panel of Table 1 presents summary statistics from this survey. We gathered information on family size, fishing experience, two personality measures, two measures of our fishermen's attitudes towards each other, and a measure of the fishermen's competitiveness. Nonpoolers come from significantly larger families than poolers $\left(p_{t}=0.02\right)$ but the mean family size of the fishermen, taken together, is not significantly different from that of the students $\left(p_{t}=0.56\right)$. Although the poolers have been fishing for shiroebi longer than the nonpoolers, the average total fishing experience of the two groups is not significantly different $\left(p_{t}=0.35\right){ }^{10}$

The cooperation scale is comprised of four statements to which respondents either agreed, disagreed or offered no response. The scale generates a score between -4 and 4 and those participants with more cooperative predispositions (e.g., they believe cooperation and reciprocity are virtuous) have higher scores. The Rotter score is based on the general scale first developed in Rotter (1966) which was constructed to capture individual differences in "locus of control." Individuals with an internal orientation, or locus of control, tend to believe that they create opportunities and that their own agency determines their fate. On the other hand, individuals with an external orientation believe in fate and that they are simply a small cog in some larger machine. In our implementation, respondents with higher Rotter scores are more internally oriented. The exact statements for each personality scale appear in Appendix B. As we anticipated, poolers have more cooperative personalities than nonpoolers but the difference is only mar ginally significant $\left(p_{t}=0.08\right)$ and the students are neither more nor less cooperatively oriented than the fishermen $\left(p_{t}=0.23\right)$. Likewise, although the poolers tend to be more internally oriented than the nonpoolers, we found no significant pair-wise difference in locus of control when comparing poolers or nonpoolers $\left(p_{t}=0.14\right)$ or when comparing students and fishermen $\left(p_{t}=0.54\right)$.

We also asked our fishermen about their conversations with other fishermen. Respondents could indicate an intimacy of discussion that ranged from rarely speaking to other fishermen to an active exchange of opinions. Most fishermen responded that they had frequent active exchanges with other fishermen and there were no significant differences between poolers and nonpoolers $\left(p_{t}=0.29\right)$. The second attitudinal question we asked the fishermen was how they considered the other fishermen. They could categorize other fishermen as competitors, strangers, friends or like family. Here we do find highly significant differences that make sense. Most poolers consider other fishermen friends and most nonpoolers consider them competitors. The mean attitude is different at better than the $1 \%$ level. Lastly, we were curious about how competitive the fishermen were towards each other. Therefore, we asked them how interested

\footnotetext{
${ }^{10}$ All the $p_{t}$-values presented in our discussion of summary statistics come from two-tailed t-tests.
} 
they were in the productivity of the other boats. It was no surprise to find that both groups were very interested and there was no significant difference in their mean interest $\left(p_{t}=0.38\right)$.

The lower panel of Table 1 lists summary statistics from the experiment. Combining all ten periods, poolers do cooperate slightly more than nonpoolers $\left(p_{t}=0.10\right)$ and, taken together, the fishermen cooperate much more than the students $\left(p_{t}<0.01\right)$. For that matter, pooled student reactions are significantly different in each of the four comparisons of experimental behavior at better than the $1 \%$ level indicating that there may be problems generalizing from student behavior to field behavior. Much of the difference in contributions between poolers and nonpoolers is the result of the social disapproval treatment. A test of the pre-disapproval contributions shows no significant difference $\left(p_{t}=0.25\right)$, but the post-disapproval contributions are highly significantly different $\left(p_{t}<\right.$ 0.01 ) which suggests that poolers were more sensitive to social disapproval than nonpoolers were. Concerning social disapproval, we find that our participants were willing to pay to show their disapproval, despite there being no material incentive to do so. Students show more disapproval than fishermen $\left(p_{t}<0.01\right)$ and nonpoolers show disapproval more frequently than poolers $\left(p_{t}=0.02\right)$, but these differences are confounded by the fact that poolers contribute more than nonpoolers and fishermen contribute more than students.

To give the reader a sense of the dynamics of the experimental behavior, Figure 1 plots the mean contribution by round in panel (a) and the frequency of social disapproval in panel (b). As hinted at by Table 1, the contribution levels of the poolers and nonpoolers are similar for the first five periods but start to bifurcate after social disapproval is instituted. Starting in round 7 pooler contribution levels begin to increase and in round 8 the nonpooler contribution levels drop dramatically. By comparison, the student contribution levels are relatively low but flat over the course of the entire experiment; however they do show a large endgame effect. We see that the student frequency of disapproval falls slightly over time, perhaps because it is obvious that it is not having an effect. Initially, the nonpoolers frequency of disapproval increases with free riding, but then the nonpoolers seem to also give up in round 8. Meanwhile, the poolers disapprove steady between 10 and 20 percent of the time.

Table 2 provides a more structured test for differences in contributions at the group level. In column (1) we regress the group's total contribution in round $t$ on indicator variables for pooling and being a student and find evidence of significant differences in both cases. However, column (1) does not account for the panel nature of the contributions data. In column (2) we add group-level random effects which increases the standard error on the pooler point estimate to the point where the coefficient is no longer significantly different from zero. Further, the random effects account for $34 \%$ of the overall variation in group contributions and the chi-squared test of whether the panel estimate is different from the pooled estimate is significant at the $1 \%$ level. In sum, pooler groups do not robustly contribute more, but student groups do contribute 6.269 coins 
less than the omitted category, the fishermen who do not pool. ${ }^{11}$

\section{Constructing Measures of Social Preference}

Our goal is to use the data from our experiment to construct measures of social preference that we can link to fishing outcomes. Perhaps the simplest measures of cooperation and the willingness to show social disapproval that one could construct would be participant means from the pooled data. However, the more nuanced explorations of social preferences have demonstrated that the reactions of participants to what other participants do or to the anticipation of what other participants will do is just as important (e.g., Charness and Rabin, 2002 or Falk et al., 2003). Simple means can not capture these reactions. To be comprehensive, we develop measures that reflect the reactions of participants to what others in his group have done and measures that account for unconditional predispositions.

Our first measure of social preference is the most obvious. For each individual we regress the person's contribution in round $t$ on the total contribution of everyone else in the group in the previous round. The resulting coefficient, which we call conditional cooperation, can take positive values meaning that the participant contributes more in reaction to demonstrated cooperativeness, it can be zero indicating that the participant follows some self-imposed "dominant strategy" (which need not be to contribute nothing), or it can be negative indicating that the participant shirks more when he expects the others to be particularly cooperative. Specifically, we define conditional cooperation to be $\beta_{i}^{C C}$ in the following regression:

$$
C_{i, t}=\beta_{i}^{0}+\beta_{i}^{C C}\left(\sum C_{-i, t-1}\right)+\epsilon_{i, t}
$$

where $C_{i, t}$ is individual $i$ 's contribution in round $t, \beta_{i}^{0}$ is an intercept, $\sum C_{-i, t-1}$ is the total contribution of the other three group members in period $t-1$, and $\epsilon_{i, t}$ is an error term. Notice that $\beta_{i}^{0}$ might be interpreted as the individual's predisposition to cooperate. For example, a participant who has a relatively low level of conditional cooperation but has a large intercept could be thought of as more altruistic. With this in mind, we define $\beta_{i}^{0}$ as a measure of individual $i$ 's unconditional cooperation. ${ }^{12}$

\footnotetext{
${ }^{11}$ The differences we find between student behavior and the behavior of non-students echo other VCM results discussed in List (2004) and Carpenter et al. (2004a) and echo the sentiment that student social preference results may only provide a lower bound.

${ }^{12}$ One alternative way to create such measures of cooperation would be to pool the data across individuals and then calculate a pooled beta. From the pooled beta, one could predict contribution levels for each individual. While this would increase the degrees of freedom of (and therefore tighten) the estimate substantially, it would also effectively defeat the purpose because the predicted values would assume the same structure of social preference for each participant. It is the heterogeneity of preferences that we are interested in. A second alternative would be to interact participant fixed effects with the lagged contributions of the other players in a pooled regression. However, this is essentially the same as what we have already done.
} 
The second measure of social preference that we consider is the propensity of each participant to show disapproval of the amount that the other three group members kept for themselves. To construct this measure, we regress whether or not participant $i$ buys an unhappy face in round $t$ on the total number of coins that the rest of the group kept in round $t$. Positive coefficients on the amount kept by the rest of the group indicate that participant $i$ is more likely to disapprove when the others keep more, a zero coefficient indicates the participant does not condition his disapproval on the allocation decisions of the others, and a negative coefficient indicates that the participant is less likely to show disapproval when the amount kept is high. The resulting linear probability model takes the following form:

$$
D_{i, t}=\alpha_{i}^{0}+\alpha_{i}^{P T D}\left(30-\sum C_{-i, t}\right)+\delta_{i, t}
$$

where $D_{i, t}$ is an indicator variable that takes the value of one when participant $i$ purchases an unhappy face, $\alpha_{i}^{0}$ is an intercept, $\alpha_{i}^{P T D}$ is individual $i$ 's propensity to show disapproval, and the last item is another error term. ${ }^{13}$

For our third measure of social preference, we redid our conditional cooperation regressions with an additional regressor, the total number of unhappy faces participant $i$ saw in the previous round. We call the coefficient on the new regressor, participant i's response to disapproval. This measure is interesting because participants can react pro- or anti-socially to collective shunning. A positive coefficient suggests that participant $i$ contributes more when the group has been shunned and a negative coefficient indicates that participant $i$ reacts spitefully to disapproval. To create our measures of $i$ 's response to social disapproval we ran the following at the individual level:

$$
C_{i, t}=b_{i}^{0}+b_{i}^{C C}\left(\sum C_{-i, t-1}\right)+\beta_{i}^{R T D}\left(\sum D_{i, t-1}\right)+u_{i, t}
$$

where the first three terms replicate the conditional cooperation regression and $\beta_{i}^{R T D}$ is our measure of $i$ 's response to the total number of unhappy faces he saw at the beginning of the round, $\sum D_{i, t-1} \cdot{ }^{14}$ As with the conditional cooperation measure, the intercept in the above regression is informative and therefore we call $b_{i}^{0}$ participant $i$ 's unconditional cooperation controlling for one's reaction to disapproval, which we abbreviate to $i$ 's unconditional response.

The five panels of Figure 2 illustrate the cumulative distributions (cdfs) of the five social preference measures. To foster comparisons, we include cdfs for poolers, nonpoolers, and students. Beginning with conditional cooperation in panel (a), it appears that students and nonpoolers are more conditionally cooperative than poolers which presents a puzzle because we expected the poolers

\footnotetext{
${ }^{13}$ In this case the intercept only really mattered when an individual's propensity to disapprove was zero which indicated that the participant either never disapproved or always disapproved. In the few cases where the participant always disapproved, we recoded the participant's propensity as one.

${ }^{14}$ The reason we did not simply use the coefficient on the lagged contributions of the other group members from this contribution regression (i.e., $b_{i}^{C C}$ ) as our measure of conditional cooperation is that this regression doesn't utilize the data from the first five rounds of the experiment.
} 
to be more cooperative. The puzzle is "solved," however, when one considers the unconditional cooperation cdfs in panel (b). The poolers have higher levels of unconditional cooperation than either nonpoolers or students.

Panel (c) indicates that close to $40 \%$ of each population never showed disapproval, but considering only positive propensities to disapprove, it appears that students are more likely to disapprove than nonpoolers or poolers when the rest of the group increases the amount of coins that they keep. Recall that we suggested that the differences in the frequency of disapproval illustrated in panel (b) of Figure 1 could be due to differences in contribution rates. It seems that this explanation is not the full story because the same differences arise when we control for the amount of free riding in the group. Considering only the negative propensities, we find that approximately $20 \%$ of students and nonpoolers are actually more likely to show disapproval when others contribute a lot. By contrast, fewer than $10 \%$ of poolers engage in such behavior.

To a large extenxt we can use the social preference cdfs in Figure 2 to explain the contribution dynamics in Figure 1 . One reason for the low contributions in the student groups (again, recall Figure 1) is that many of the students did not respond prosocially to disapproval. As one can see in panel (d) of Figure 2, more than half of the students had zero response to disapproval coefficients. The incidence of zeros is far lower among the fishermen. Only $10 \%$ of the poolers and $15 \%$ of the nonpoolers do not respond. However, of more interest is the fact that most of the poolers have positive response to punishment coefficients while $70 \%$ of the nonpoolers have negative reactions to social disapproval. This suggest that the reason that contributions fall among nonpoolers and rise among poolers is that nonpoolers respond spitefully to social disapproval while poolers take it as a signal to contribute more. As is the case when one compares panels (a) and (b) the constants in the response to disapproval regressions (panel (e)) are mirror images of the coefficients on the lagged disapproval cdfs in panel (d). ${ }^{15}$

\section{Is there a Treatment Effect of Pooling on So- cial Preferences?}

Before addressing our main hypothesis, that social preferences can partially explain the variation in fishing productivity, we will discuss a related issue motivated by the discussion of endogenous preferences in Bowles (1998): are any of the differences that we saw in the distributions of social preference due to exposure to the pooling institution. While it would be interesting to know what portion of the differences are due to treatment and what portion are due to selection, because our participants have been fishing for some time it is impossible to accurately assess any selection effects. That is, to properly identify selection

\footnotetext{
${ }^{15}$ We do not report test statistics for differences in the social preference cdfs. Controlled (parametric) tests are performed in the next section and indicate that all the differences we discussed above are statistically significant.
} 
effects we would need to assess the impact of social preferences (at their level when the individual begins fishing) on the decision of what boat to join, but such an analysis is impossible because only 7 fishermen have less than 15 years of experience and they all choose to pool. ${ }^{16}$ That said, there are also institutional reasons to think selection is not much of an issue here.

The poolers and their predecessors have been fishing for shiroebi since the 18 th century. The pooling arrangement, spurred by the victory over the wholesalers, came about in the 1960s as a best response to the economic conditions of fishing at the time. Specifically, the pooling arrangement allowed the fishermen to price discriminate among the buyers and therefore pooling arose as an economic choice of all the people fishing in the 1960s, rather than some process of differential selection into (or away from) pooling by some due to their social preferences. Part of the reason why the pooling arrangement has been stable is that most skippers simply adopt whatever compensation scheme their fathers used. Therefore, unless social preferences are completely inherited from one's father (who as we already suggested adopted pooling for economic not personality reasons), we do not expect many new fishermen to sort into jobs based on their social preferences.

Without much cause to worryabout selection, we can take advantage of our survey to conduct a very straightforward test for treatment effects. Specifically, if social preferences are acquired (or maintained) differentially on pooling boats than on nonpooling boats, then we should see a correlation between years of experience with pooling and the levels of social preference.

Table 3 reports the results of our treatment analysis for each of the five social preference measures. Because the scales of the social preference measures vary so much we report standardized regression coefficients. ${ }^{17}$ Overall, we see that controlling for experience, whether or not the individual is a boat skipper, our two personality measures which should further assuage readers concerned with selection, and our two measures of associations, there are significant differences in the levels of social preference between poolers and non-poolers. As hinted at in Figure 2, poolers are less conditionally cooperative $(p<0.05)$, but more unconditionally cooperative $(p<0.05)$; poolers have lower propensities to punish $(p<0.001)$, respond significantly more prosocially to social disapproval $(p<0.10)$, and have lower unconditional responses to disapproval $(p<0.05)$.

We also find that experience is correlated with three of the five social preference measures. The baseline effect of experience is to reduce conditional cooperation, increase unconditional cooperation, and reduce one's propensity to show disapproval, but experience does not appear to affect one's reaction to social disapproval. In general, two of the reactive measures of social preference,

\footnotetext{
${ }^{16}$ Despite the confounding effect of experience, we did explore selection by regressing whether an individual was on a pooling boat or not on the five measures of social preference and found that none of the coefficients were significant. If one assumes that social preferences are stable (an assumption we are not prepared to make), then we have found no evidence of selection.

${ }^{17}$ That is, the interpretation of the coefficients in Table 3 (and Table 4 ) is the number of standard deviations that the dependent variable changes when the independent variable increases by one standard deviation.
} 
conditional cooperation and the propensity to disapprove, seem to decay with experience. At the same time, however, we also find significant treatment effects of pooling that counteract the overall decay of these two reactive measures of social preference. The differential effect of pooling on conditional cooperation is positive $(p<0.05)$, the effect on unconditional cooperation is negative $(p<0.05)$, the effect on the propensity to disapprove is positive $(p<0.01)$, and the coefficients on these interactions almost completely counteract the baseline effects. In other words, we have uncovered evidence that social preferences are endogenous: the pooling institution attenuates the natural erosion of two of our three reactive measures of social preference.

There are two other interesting facts that arise from our examination of treatment effects. First, we find that boat skippers are different in interesting ways. Skippers are more conditionally cooperative $(0.57$ standard deviations more than crew members), less unconditionally cooperative (0.48 standard deviations less), show higher propensities to disapprove ( 0.86 standard deviations higher), and are less responsive to disapproval (0.55 standard deviations less). In other words, boat captains are more willing to cooperate when their crew does, are not hesitant to inform the crew that free riding will not be tolerated, but react more spitefully when they are publicly shamed.

The second ancillary finding uncovered in Table 3 is that the Rotter score is correlated with our social preference measures. Those fishermen who are most internally-oriented are less conditionally cooperative, have higher propensities to disapprove, and are more unconditionally responsive to social disapproval. These correlations will become more useful in our analysis of fishing productivity.

\section{Do Social Preferences Belong in the Produc- tion Function?}

In this section we examine the links between social preferences and fishing productivity. We have two goals; first, we seek to make a general methodological point. Demonstrating that social preferences have a direct effect on worker productivity provides a much needed link between the extensive laboratory literature on social preferences and the economic mainstream. Our second, more modest, goal is to test if the productivity gains accruing to poolers are due entirely to structural factors (e.g., pair-fishing) or if there is a differential prosocial effect of social preferences on the work effort of pooling fishermen that explains part of this productivity gap.

As mentioned briefly in section 2, we gathered haul data from 115 trips into the bay by the 12 boats in the two groups. There is considerable variation in the amount of shiroebi that boats return to harbor with. For example, we witnesses eight instances where the boats caught nothing. Overall however, the mean catch per unit effort is 89.94 kilos with a standard deviation of 75.54 kilos.

We estimated a series of fishing production functions and report the results 
in Table 4. Included in our estimates are all the important factors of production (informed by our discussion with skippers and our experience aboard a number of the boats) that actually vary significantly between poolers and nonpoolers. ${ }^{18}$ First, we include the boat skipper's years of experience as a skipper. It takes many years to learn the seabed, where the best fishing spots are (something that can vary by season), and how best to manage your crew. Therefore, we anticipated that boats with skippers who have considerable experience would be more productive. At the same time, young skippers may be more amenable to, and adept at, taking advantage of new technologies, even thought they may not be as skilled in the basics as the older skippers. With both of these rationales in mind, we hypothesized that the effect of the Skipper's Experience might be concave which caused us to include Skipper Experience ${ }^{2}$. For the same reasons, we expected that the average experience of the crew would matter. ${ }^{19}$ The horse power of the boat's engine is important because it determines the size of the net that can be used, the speed of trawling, the extent of the search, and the speed back to port. To capture the structural factors that make poolers more productive, we included the indicator variable, Pool Revenues.

All our estimates utilize the Tobit estimator because some boats return to port without any fish. Since our social preferences are measured on such different scales (see Figure 2), we report standardized regression coefficients throughout to make our point estimates more comparable. To control for un-modeled weather or demand factors, we included fishing week fixed effects in each regression. ${ }^{20}$ We also we explored including boat random effects but did not add them because the panel variance component in the basic production function was not important (i.e., $5 \%$ of the total).

As one can see from Column (1) of Table 4, fishing productivity does appear to be concave in skipper experience $(p<0.05$ for the linear term and $p<$ 0.10 for the squared term). In fact, using the non-standardized coefficients, our estimate suggests that skippers with 36 years of experience are the most productive captains. The mean crew experience, on the other hand, does not seem to matter significantly, although the coefficient is positive. ${ }^{21}$ As expected, the horse power of the boat is positively associated with the amount of fish caught $(p<0.01)$. Finally, controlling for all the other important factors of production, we see that poolers tend to catch 0.404 standard deviations (or 30 kilos) more than nonpoolers $(p<0.01)$.

\footnotetext{
${ }^{18}$ For example, both poolers and nonpoolers use the same sort of net and therefore this factor is controlled for naturally.

${ }^{19}$ This intuition did not seem to be correct, as one can see from Table 4, the coefficient on mean crew experience is significantly different from zero only once and when we tried the squared term it added nothing to the analysis.

${ }^{20}$ We can not use fishing day fixed effects because poolers and nonpoolers alternate fishing days and therefore the fixed effects would be perfectly correlated with our pooling indicator variable. We also can not use bost fixed effects because our vector of controls does not change over the period of observation and therefore boat fixed effects would be highly correlated with these unchanging boat characteristics.

${ }^{21}$ We also tried using the standard deviation of the crew experience instead, but it did not predict any better.
} 
In columns (2) through (4) we add the boat mean values of our social preferences to the basic production function. We add our reactive measures one at a time since they are highly correlated with each other. We also add our two unconditional measures at the same time as their respective reactive measures because we are interested in whether the reactive measures predict when we control for unconditional predispositions. Overall, we see that adding social preferences improves the estimates (i.e., the log likelihoods are noticeably higher), the direct effect of each social preference on productivity is significant, and the interactions of social preference and pooling soak up some of the variance in productivity that had been attributed to the pooling indicator variable in column(1). This last fact suggests that part of the reason pooling boats are more productive is because social preferences matter.

More specifically, in column (2) we see that the baseline effect of conditional cooperation on all boats is associated with a 0.625 standard deviation increase in productivity $(p<0.01)$ and a further 0.569 standard deviation increase for poolers $(p=0.12)$; however, the interaction term is not significant at conventional levels. At the same time, unconditional cooperation does not appear to be robustly related to productivity even though the interaction term is large and close to significant. It is also interesting that the pooling indicator variable is no longer significantly different from zero although the point estimate has not changed. This suggests that the differential effect of conditional cooperation partially explains why poolers are more productive.

The results listed in column (3) indicate that the propensity to show disapproval when the others shirk is associated with a 0.340 standard deviation increase in productivity $(p<0.01)$ and a further increase of 0.435 standard deviations that accrues only to poolers $(p<0.10)$. In this case the addition of social preference causes the pooling indicator to lose both significance and magnitude. This is further evidence that social preferences matter in general, and in particular for poolers.

Interesting, the baseline effect of one's response to social disapproval (column (4)) is negative indicating that those boats with participants that responded more prosocially to disapproval actually produce less $(p<0.10)$. However this result must be tempered by the fact that the pooling interaction coefficient is positive and of the same magnitude indicating that the effect is attenuated for poolers. The results are very similar for one's unconditional response to disapproval. Further, the pooling indicator again loses significance and shrinks somewhat.

The results in columns (1) through (4) are very encouraging, but how robust are they? For example what if we do not assume that social preferences are exogenous. In the previous section we argued that social preferences change as the fishermen gain both general experience and specific experience with pooling. It therefore can not be too hard to think of scenarios in which there is feedback, for example, from productivity to social preference. In columns (5) through (7) we control for this possibility by instrumenting for our social preference measures.

Luckily our survey provided us with a number of variables that are highly 
correlated with social preference but should be exogenous with respect to productivity. One measure, highlighted in the previous section, is the Rotter score. The Rotter score is highly correlated with all our social preference measures (although it only shows up significantly in three of the regressions in Table 3) and therefore it must also be correlated with productivity. However, many previous studies have found no link between locus of control and productivity (e.g., Guion and Gibson, 1965; Szilagyi et al., 1976; Johnson et al., 1984; Hollenbeck and Whitener, 1988; Blau, 1993). This suggests that the locus of control works through social preference in our implementation. ${ }^{22}$ We also used other variables listed in Table 1 such as family size as instruments. Most of these variables are not too hard to defend as instruments. For example, it is not obvious why family size would affect productivity directly if one realizes that the fishermen do not actually consume any of the fish that they catch nor is it clear that fishing productivity will have a large effect on one's family size, especially for those unmarried crew members who still live at home or those who have adopted fishing in retirement.

The instrumented production function estimates in columns (5) through (7) actually reflect a stronger case for the importance of social preferences. Now all the baseline effects of social preference and all the pooling interactions effects are statistically significant. In column (5) we see that conditional cooperation improves efficiency overall, but poolers benefit 0.819 standard deviations more than nonpoolers, and unconditional cooperation has a significant positive effect on productivity, but only for poolers. Column (6) is very similar to column (3) in that it indicates that the propensity to disapprove is associated with productivity gains, but now the differential effect on poolers has increased from 0.435 standard deviations to 0.685 standard deviations. Further, in column (7) we see that the response to disapprove remains negatively associated with productivity among nonpoolers, but the new estimate of the coefficient on the pooling interaction is significant and positive. In fact, the sum of the baseline and differential effects suggest that the response to punishment is, indeed, positively associated with productivity for the poolers.

It is also important to note that each of the pooling indicator variables in regressions (5) through (7) lose statistical significance and magnitude when the instrumented social preference measures are included. Therefore, we continue to see that the differential impact of social preferences on the pooling boats accounts for a substantial amount of their productivity advantage. Lastly, in the bottom panel of Table 4 we report the Hausman test $p$-values from the comparisons of the coefficients in the instrumented and un-instrumented models. These $p$-values suggest that endogeneity might have been a problem in two of the three cases: conditional cooperation and the response to social disapproval.

\footnotetext{
${ }^{22}$ Additionally, because the test-retest reliability of the Rotter scale is relatively high we can assume this personality measure is relatively stable.
} 


\section{Concluding Remarks}

To summarize our results, we find that student behavior in our voluntary contribution experiment lacks external validity with respect to our second subject pool, fishermen who face the sort of social dilemma modeled by our experimental protocol in their daily work lives. Specifically, we find that fishermen are significantly more cooperative than students - a result that has become a "stylized fact" of the field experimental literature. Our second significant finding is that three of our five experimental measures of social preference (conditional and unconditional cooperation, and response to social disapproval) are endogenous to the adoption of a cooperative institution, under which boats pool their fishing efforts. We find that pooling experience attenuates a general decay of social preference that affects all fishermen. Our third result is the most important: we find that experimental measures of social preference can be directly linked to fishing productivity. Those crews that exhibit greater degrees of conditional cooperation and the propensity to disapprove of shirking are more productive. However, the baseline affect of being more responsive to the disapproval of others appears to be a drag on productivity. Lastly, we have found that social preferences contribute significantly to explaining why pooling boats are more productive than their nonpooling competitors. The poolers catch more, not only because of institutionalized cooperative fishing techniques, they catch more because their stock of social preferences has a differential effect on their efforts.

Our results can also be viewed as one of the few clean tests of Rotemberg's hypothesis that altruism should evolve under team production and increase productivity (1994:688). Recall our measure of unconditional cooperation, $\beta_{i}^{0}$, measures how much one would contribute even if all the other participants contributed zero. In the context of team production, this is clearly the sort of altruism modelled by Rotemberg. Indeed, Figure 2 (panel (b)) and Table 3 (column 2) confirm this hypothesis. Our poolers, who are exposed to a second level of team production, are significantly more altruistic towards each other than the nonpoolers are. Further, Table 4 indicates that the poolers are more productive, largely because of their social preferences, including altruism.

While simply finding a link between social preferences and productivity goes a long way to bridging the gap between experiments and the economics mainstream, it is also important to assess the magnitude of the social preference effects. Recall that the mean catch per unit of effort among the fishermen is approximately 90 kilos. Poolers typically catch 0.4 standard deviations, or 30 kilos, more. The base line effect of a standard deviation increase in conditional cooperation is to increase the catch of all boats by 47 kilos. However, the differential effect of conditional cooperation on poolers is an additional 43 kilos. Similar results for the other social preference measures can be calculated from the standardized regression coefficients in Table 4. The point, however, is that social preferences do not only matter for the productivity of our fishermen, they matter a lot. 


\section{Appendix A: Experiment Instructions (back- translations from Japanese)}

Thank you very much for participating in the experiment today. You will be asked to repeat the exercise 10 times. After that we request that you fill out a brief questionnaire.

In the experiment, you will earn money. The amount you will earn depends on the decisions you and everyone else make during the experiment. This experiment aims to study individual behavior when money is at stake, thus we will use real money during the experiment. You will keep any money that you earn during the experiment.

Any decisions you make in the exercises or responses you give in the questionnaire will be strictly confidential. We will never tell anyone your responses or choices. To assure your responses are confidential, we ask you to not speak to each other until the entire experiment is completed.

\section{Rules of the exercise:}

Please check if you have the following items are in front of you: A pencil, instructions (this paper), and a large brown envelop.

The exercise will be repeated 10 times but a new rule will be applied for the last 5 rounds. We will explain new rule before the 6 th round.

You are a member of a group of four people: there are three other people in the group with you. You will all stay in the same group for each of the 10 rounds.

To understand the exercise, imagine yourself in a situation where you have to decide how to allocate resources intended for fishing. You may allocate your money to buy new fishing equipment or to build a lighthouse. If you buy new equipment, you will be able to increase your catch and income. If a lighthouse is built, it will increase the safety and seawothiness of every boat regardless of whether they allocate money for building the lighthouse or not.

In the exercise, you will be asked to decide how much of your money to keep and how much to allocate towards a group project.

At the beginning of each round, we will give you a white envelope with ten 50 yen coins inside. Each person in the group will then decide privately how many of these 10 coins to allocate to the group project and how many to keep for himor herself. Everyone in the group benefits equally from the money allocated to the group project, but only you benefit from the money you keep. Please put the money you would like to keep in the brown envelop and return the rest that you would like to allocate to the group project in the white envelop. We will collect the white envelope. Please keep the brown envelop with you during the experiment.

When all four members of the group have decided how many coins to allocate to the group project, we will add up all the money from the four group members. When we know the total, we will double it. Each person will then receive an equal share of the doubled amount. Each person also keeps what ever money 
he or she puts in the large brown envelope.

Here is an example to illustrate how the experiment works. Each person decides how much to allocate to the group project privately, so you will not know what anyone else has decided when you make your choice. Suppose that in the first round everyone in your group, including yourself, allocate all 10 coins to the group project. In total there are $10+10+10+10=40$ coins in the group project. The group project will double this amount which makes the total 80 coins. Each of you then receives an equal share, i.e., 20 coins.

To continue the example, let us now suppose in the second round. Everyone in the group receives another 10 coins at the beginning of the round. Imagine that this time everyone in the group contributes no money to the group project. In total there are $0+0+0+0=0$ coin in the group project. Because nobody contributes to the group project, nobody receives anything from the group project. Each person's earnings for round two are just the 10 coins that each person kept.

Let's continue the example for one more round. Now say that you allocate nothing to the group project and the other three people in the same group allocate everything to the group project. The group project will receive a total of $0+10+10+10=30$ coins. We double this amount which makes the total of 60 coins. Each person receives an equal share of the 60 coins, i.e., 15 coins from the group project.

In the round 3 you would earn total of 25 coins ( 15 from the group project plus the 10 you kept), while the other members will earn 15 coins from the project.

After each round, you will be provided with a report that contains all four members' allocations to the group project in random order, the total number of coins allocated to the project, and your share of income from the group project. Please note that group members' allocations are written in random order so that it is impossible to identify what any individual allocated to the project.

The above is only an example. You will play 5 rounds and each of you will decide, on your own, how to allocate the 10 coins that you receive at the beginning of each round.

Are there any questions about how the experiment will proceed?

\section{Rules for exercise 2 (only to be handed out after exercise 1 has been completed)}

Exercise 2 is very similar to exercise 1 , but there will be one difference in the procedures.

The first part of each decision making round will be exactly the same as exercise 1 . There will be 5 decision making rounds and you will each receive 10 coins at the beginning of each round. You will decide privately how much money to allocate to the group project and how much to keep. When everyone in the group has made this decision, we will calculate the total contribution. We will then double the total contribution. Each person will receive an equal share of the doubled amount. 
The only difference between exercise 1 and exercise 2 is that you will have the possibility to send a message to the rest of your group. This is the message you can send:

\section{show "unhappy face"}

You will be asked to fill out an order form for an "unhappy face" after each round. We will collect the order forms and count the number of "unhappy faces" ordered in each group. At the beginning of the next round, the white envelop that you receive will have as many unhappy face marks as the totoal number of marks ordered by all your group members.

Please note that ordering the "unhappy mark" will cost you 10 yen. When you see stamps on your envelop, you know that some of the group members have spent 10 yen each to let the rest of the group know that they are unhappy with the amount that was allocated to the group project. Anyone who decides to send this message will do so anonymously. Nobody will know who sent the messages.

Are there any questions about how the exercise will proceed?

\section{Appendix B: Data Definitions}

The Cooperation scale statements were: 1) "It is virtuous to compete;" 2) "It is virtuous to cooperate;" 3) "One must reciprocate kindness;" 4) "People should revenge wrongs done to them." One's score increased by one for agreeing with 2 and 3 and for disagreeing with 1 and 4 . One's score decreased by one for agreeing with 1 and 4 and disagreeing with 2 and 3 .

The Rotter score statements were: 1) "I believe my success depends on ability rather than luck;" 2) "I dislike taking responsibility for making decisions;" 3) "I make decisions and move on;" 4) "I believe that unfortunate events occur because of bad luck;" 5) "I like to take responsibility for making decisions;" and 6) "I tend to analyze situations too much and therefore miss opportunities." Agreeing with statements 1, 3, and 5, and disagreeing with 2, 4, and 6 increased one's score by one point. Agreeing statements 2, 4, and 6, and disagreeing with 1,3 , and 5 reduced one's score by one point.

Meet Other Fishermen is a likert scale response to the question "What do you do when you meet your fellow fishers?" Responses to this question include: 1) "I often speak about myself;" 2) "I tend to listen to them;" 3) "I actively exchange opinions with them;" and 4) "I rarely have conversations with them". Scores of zero to three were assigned to the statements $4,1,2$, and 3 , respectively.

Consider Other Fishermen is a likert scale response to the question "How do you consider your fellow fishers?" Alternative responses to this questions were: 1) "I consider my fellow fishers as family members;" 2) "I consider them as friends;" 3) "I consider them as strangers," and 4) "I consider them as competitors." 
Scores of two, one, zero and minus one were assigned to the statements 1, 2, 3, and 4 , respectively.

Interest in Other's Catch is one's responses to the question, "At the end of a fishing day, how keen are you to know how much the other fishers catch?" The responses are measured on a likert scale: scores of three, two, one and zero were assigned to the responses, "very much;" "a little;" "not very much;" "not at all," respectively.

\section{Tables and Figures}

\begin{tabular}{|c|c|c|c|}
\hline & $\begin{array}{l}\text { Pooler } \\
(\mathrm{N}=14)\end{array}$ & $\begin{array}{c}\text { Nonpooler } \\
(\mathrm{N}=13)\end{array}$ & $\begin{array}{l}\text { Student } \\
(\mathrm{N}=26)\end{array}$ \\
\hline \multicolumn{4}{|l|}{ Demographics, Personality and Attitudes } \\
\hline Family Size & $3.78(1.37)$ & $5.61(2.26)$ & $4.92(0.98)$ \\
\hline Fishing Experience & $21.86(20.56)$ & $28.15(10.72)$ & - \\
\hline Cooperation Scale & $1.07(0.83)$ & $0.38(1.12)$ & $0.38(1.13)$ \\
\hline Rotter Score & $0.78(1.62)$ & $-0.23(1.88)$ & $0.61(2.02)$ \\
\hline Meet Other Fishermen (quality of conversation) & $2.57(0.65)$ & $2.25(0.87)$ & \\
\hline Consider Other Fishermen (-1:Competitor, 0:Stra & & & \\
\hline 1:Friend, 2:Family) & $0.71(0.99)$ & $-0.58(0.79)$ & - \\
\hline Interest in Other's Catch (0:None to $3:$ Much) & $2.23(0.44)$ & $2.00(0.82)$ & - \\
\hline \multicolumn{4}{|l|}{ Behavior } \\
\hline Overall Contribution & $5.14(1.67)$ & $4.59(2.02)$ & $3.15(1.40)$ \\
\hline Pre-Disapproval Contribution & $5.07(1.74)$ & $4.80(2.24)$ & $3.33(1.71)$ \\
\hline Post-Disapproval Contribution & $5.21(2.11)$ & $4.38(1.94)$ & $2.96(1.57)$ \\
\hline Social Disapproval & $0.12(0.19)$ & $0.31(0.34)$ & $0.42(0.98)$ \\
\hline
\end{tabular}

Note: (standard deviations) 
Table 2: Are there Group Level Differences in Contributions?

\begin{tabular}{lcc}
\multicolumn{3}{c}{ (dependent variable is group total contribution in period t) } \\
\hline \multirow{3}{*}{ Pooler } & $(1)$ & $(2)$ \\
& 2.950 & 2.261 \\
Student & {$[1.388]^{* *}$} & {$[2.617]$} \\
& -5.925 & -6.269 \\
Intercept & {$[1.230]^{* * *}$} & {$[2.429]^{* * *}$} \\
& 18.325 & 18.592 \\
Group Level Random Effects? & {$[0.982]^{* * *}$} & {$[1.908]^{* * *}$} \\
rho, p-value & No & Yes \\
Log Likelihood & -486.725 & -467.865 \\
Observations & 150 & 150 \\
\hline Note: Both regressions are Tobits; & standard errors; ${ }^{* * *}$ indicates significant \\
at $1 \%, * *$ & $5 \%$, and $* 10 \%$.
\end{tabular}




\begin{tabular}{|c|c|c|c|c|c|}
\hline & Conditional & Unconditional & Propensity to & Response to & Unconditional \\
\hline & Cooperation & Cooperation & Disapprove & Disapproval & Response \\
\hline \multirow[t]{2}{*}{ Pooler } & -1.169 & 1.086 & -1.862 & 1.323 & -1.260 \\
\hline & {$[0.483]^{* *}$} & {$[0.460]^{* *}$} & {$[0.446]^{* * * *}$} & {$[0.653]^{*}$} & {$[0.598]^{* *}$} \\
\hline \multirow[t]{2}{*}{ Fishing Experience } & -1.416 & 1.437 & -2.209 & 1.268 & -0.798 \\
\hline & {$[0.577]^{* *}$} & {$[0.549]^{* *}$} & {$[0.533]^{* * *}$} & {$[0.780]$} & {$[0.712]$} \\
\hline \multirow[t]{2}{*}{ Pooler $\times$ Experience } & 1.306 & -1.270 & 2.172 & -0.971 & 0.883 \\
\hline & {$[0.608]^{* *}$} & {$[0.579]^{* *}$} & {$[0.562]^{* * * *}$} & {$[0.822]$} & {$[0.752]$} \\
\hline \multirow[t]{2}{*}{ Skipper } & 0.571 & -0.482 & 0.860 & -0.551 & 0.259 \\
\hline & {$[0.214]^{* *}$} & {$[0.204]^{* *}$} & {$[0.198]^{* * *}$} & {$[0.290]^{*}$} & {$[0.265]$} \\
\hline \multirow[t]{2}{*}{ Rotter Score } & -0.388 & 0.204 & 0.585 & -0.303 & 0.672 \\
\hline & {$[0.190]^{*}$} & {$[0.181]$} & {$[0.176]^{* * *}$} & {$[0.257]$} & {$[0.235]^{* * *}$} \\
\hline \multirow[t]{2}{*}{ Cooperation Scale } & 0.007 & 0.001 & -0.072 & 0.080 & 0.038 \\
\hline & {$[0.167]$} & {$[0.159]$} & {$[0.154]$} & {$[0.226]$} & {$[0.207]$} \\
\hline \multirow[t]{2}{*}{ Consider Fishermen } & -0.068 & 0.226 & -0.127 & -0.293 & 0.188 \\
\hline & {$[0.183]$} & {$[0.174]$} & {$[0.169]$} & {$[0.247]$} & {$[0.226]$} \\
\hline \multirow[t]{2}{*}{ Meet $\mathrm{F}$ ishermen } & 0.090 & -0.100 & -0.614 & 0.011 & 0.135 \\
\hline & {$[0.183]$} & {$[0.175]$} & {$[0.169]^{* * *}$} & {$[0.248]$} & {$[0.227]$} \\
\hline Adjusted $\mathrm{R}^{2}$ & 0.42 & 0.32 & 0.57 & 0.03 & 0.28 \\
\hline Observations & 25 & 25 & 25 & 25 & 25 \\
\hline
\end{tabular}




\begin{tabular}{|c|c|c|c|c|c|c|c|}
\hline & (1) & (2) & (3) & (4) & (5) & $(6)$ & (7) \\
\hline \multicolumn{5}{|c|}{ Production } & \multicolumn{3}{|c|}{ Froduction Function and Instrumented Social } \\
\hline & Function & Froduction & ction and $\mathrm{So}$ & Freferences & & Preferences & \\
\hline \multirow[t]{2}{*}{ Skipper's Experience (years as skipper) } & 0.973 & 2.390 & 2.147 & 1.663 & 2.855 & 2.990 & 1.895 \\
\hline & {$[0.438]^{* *}$} & {$[0.632]^{* * *}$} & {$[0.572]^{* * *}$} & {$[0.800]^{* *}$} & {$[0.691]^{* * *}$} & {$[0.987]^{* * *}$} & {$[0.695]^{* * *}$} \\
\hline \multirow[t]{2}{*}{ Skipper Experience ${ }^{2}$} & -0.830 & -1.931 & -1.577 & -1.391 & -2.241 & -2.339 & -1.606 \\
\hline & {$[0.455]^{*}$} & {$[0.549]^{* * *}$} & {$[0.483]^{* * *}$} & {$[0.748]^{*}$} & {$[0.588]^{* * *}$} & {$[0.821]^{* * *}$} & {$[0.658]^{* *}$} \\
\hline \multirow[t]{2}{*}{ Crew Experience (boat mean) } & 0.138 & 0.252 & -0.285 & 0.370 & -0.281 & -0.347 & 0.319 \\
\hline & [0.119] & {$[0.228]$} & {$[0.241]$} & {$[0.253]$} & {$[0.372]$} & {$[0.266]$} & {$[0.205]^{+}$} \\
\hline \multirow[t]{2}{*}{ Boat Horse Fower } & 0.384 & 1.100 & 0.473 & 0.566 & 0.986 & 0.602 & 0.557 \\
\hline & {$[0.144]^{* * *}$} & {$[0.278]^{* * *}$} & {$[0.162]^{* * *}$} & {$[0.160]^{* * *}$} & {$[0.268]^{* * *}$} & {$[0.211]^{* * *}$} & {$[0.161]^{* * *}$} \\
\hline \multirow[t]{2}{*}{ Fool Revenues } & 0.404 & 0.403 & 0.258 & 0.366 & -0.510 & 0.225 & 0.395 \\
\hline & {$[0.151]^{* * *}$} & {$[0.419]$} & {$[0.209]$} & {$[0.238]$} & {$[0.732]$} & {$[0.224]$} & {$[0.236]^{*}$} \\
\hline \multirow[t]{2}{*}{ Conditional Cooperation (boat mean) } & & 0.625 & & & 0.676 & & \\
\hline & & {$[0.292]^{* *}$} & & & {$[0.265]^{* * *}$} & & \\
\hline \multirow[t]{2}{*}{ Unconditional Cooperation (boat mean) } & & 0.172 & & & 0.383 & & \\
\hline & & {$[0.289]$} & & & {$[0.248]^{+}$} & & \\
\hline \multirow[t]{2}{*}{ Fropensity to Disapprove (boat mean) } & & & 0.340 & & & 0.359 & \\
\hline & & & {$[0.107]^{* * *}$} & & & {$[0.117]^{* * *}$} & \\
\hline \multirow[t]{2}{*}{ Response to Social Disapproval (boat mean) } & & & & -0.276 & & & -0.294 \\
\hline & & & & {$[0.154]^{*}$} & & & {$[0.151]^{* *}$} \\
\hline \multirow[t]{2}{*}{ Unconditional Response (boat mean) } & & & & -2.207 & & & -2.127 \\
\hline & & & & {$[0.935]^{* *}$} & & & {$[0.921]^{* *}$} \\
\hline \multirow[t]{2}{*}{ Fool $\times$ Conditional Cooperation } & & 0.569 & & & 0.819 & & \\
\hline & & {$[0.350]^{+}$} & & & {$[0.445]^{*}$} & & \\
\hline \multirow[t]{2}{*}{ Fod $\times$ Unconditional Cooperation } & & 0.818 & & & 1.623 & & \\
\hline & & {$[0.517]^{+}$} & & & {$[0.819]^{* *}$} & & \\
\hline \multirow[t]{2}{*}{ Fool $\times$ Propensity to Disapproval } & & & 0.435 & & & 0.685 & \\
\hline & & & {$[0.242]^{*}$} & & & {$[0.378]^{*}$} & \\
\hline \multirow[t]{2}{*}{ Pool $\times$ Response to Disapproval } & & & & 0.255 & & & 0.329 \\
\hline & & & & {$[0.215]$} & & & {$[0.182]^{*}$} \\
\hline \multirow[t]{2}{*}{ Fool $\times$ Unconditional Response to Disapproval } & & & & 1.870 & & & 1.840 \\
\hline & & & & {$[0.918]^{* *}$} & & & {$[0.883]^{* *}$} \\
\hline Includes fishing week fixed effects: & Yes & Yes & Yes & Yes & Yes & Yes & Yes \\
\hline Log Likelihood & -157.342 & -149.359 & -150.860 & -149.882 & -149.285 & -140.955 & -149.963 \\
\hline Hausman Test p-value & & & & & 0.01 & 0.49 & 0.05 \\
\hline Observations & 115 & 115 & 115 & 115 & 115 & 115 & 115 \\
\hline
\end{tabular}




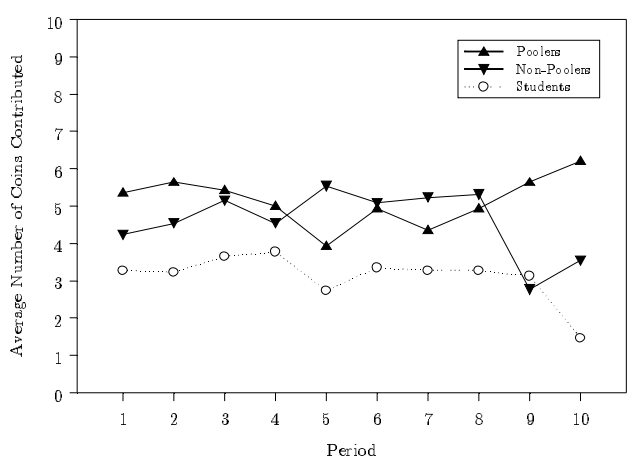

(a) Contributions

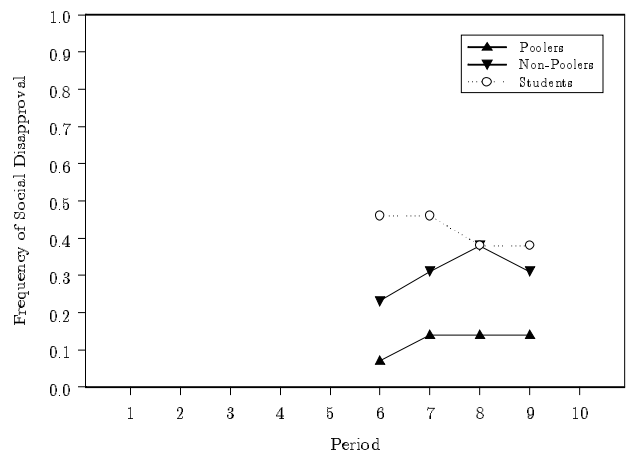

(b) Social Disapproval Figure 1: Contribution and Disapproval Time Paths. 


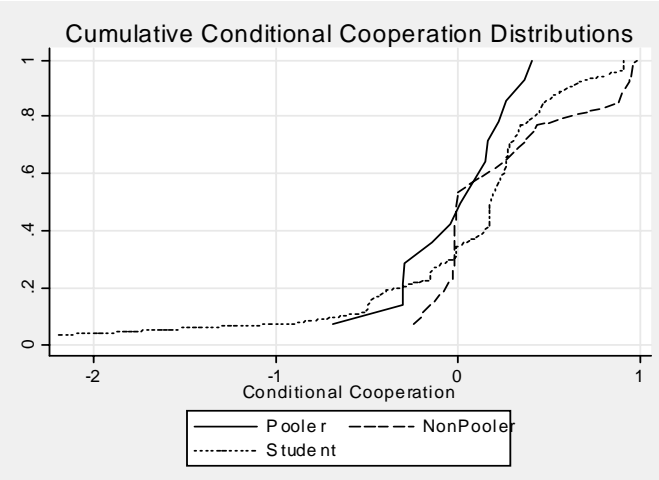

(a) Conditional Cooperation

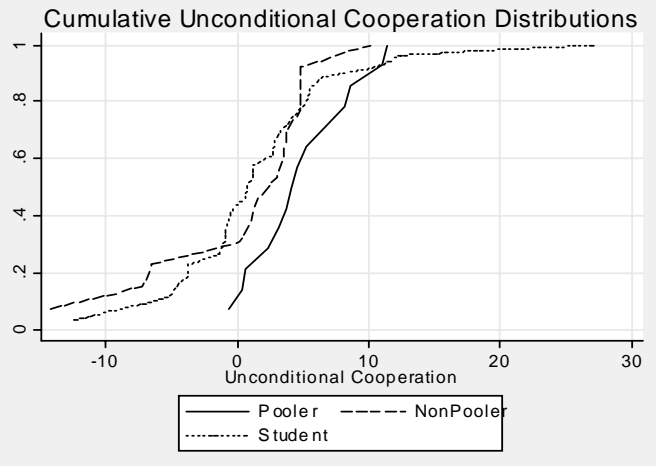

(b) Unconditional Cooperation

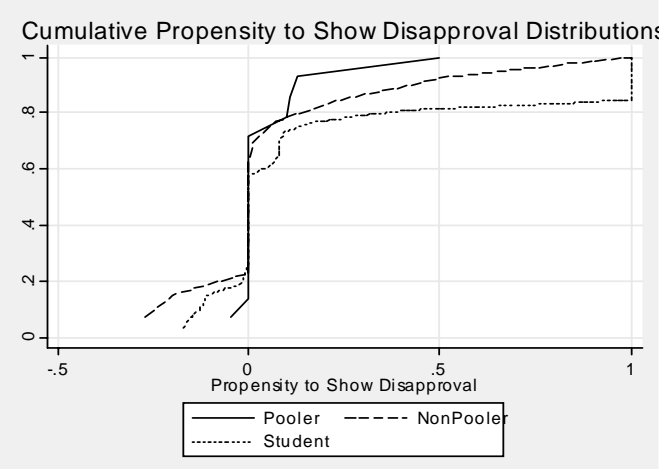

(c) Propensity to Disapprove

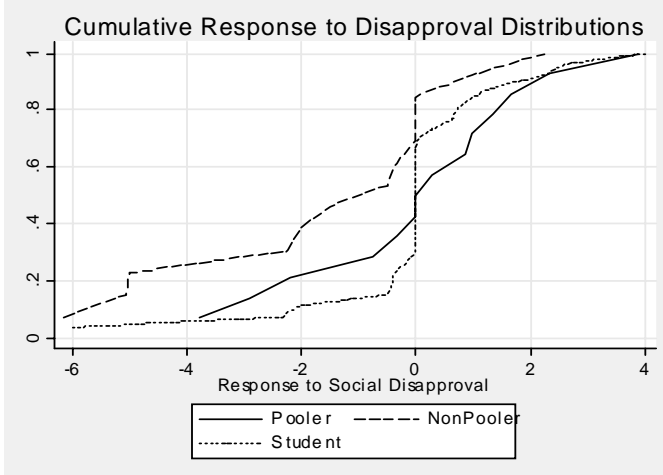

(d) Response to Disapproval

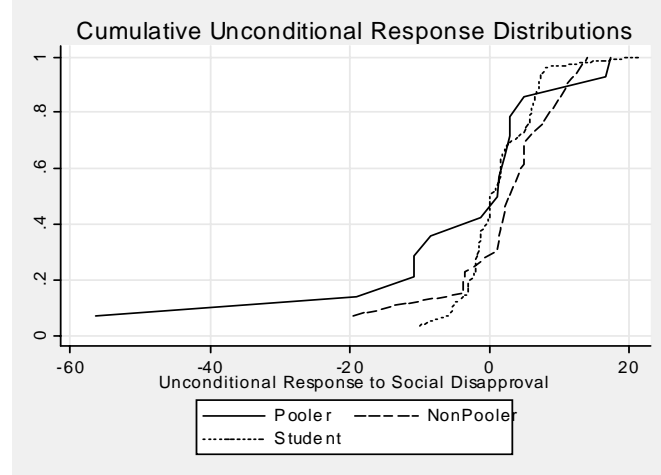

(e) Unconditional Response

Figure 2: Cumulative Distributions of Social Preferences. 


\section{References}

Barr, A., Serneels, P., 2004. Wages and reciprocity in the workplace. Oxford University Centre for the Study of African Economies Working Paper.

Blau, G., 1993. Testing the relationship of locus of control to different performance dimensions. Journal of Occupational and Organizational Psychology. $66,125-138$.

Bowles, S., 1998. Endogenous preferences: The cultural consequences of markets and other economic institutions. Journal of Economic Literature. 36, $75-111$.

Cardenas, J.C., Carpenter, J., 2005. Three themes on field experiments and economic development. In: Carpenter, J., List, J., Harrison, G. (Eds.), Field experiments in economics, JAI Press, Greenwich, pp. 71-124.

Cardenas, J.C., Stranlund, J., Willis, C., 2000. Local environmental control and institutional crowding-out. World Development. 28, 1719-1733.

Carpenter, J., 2004. Punishing free-riders: How group size affects mutual monitoring and the provision of public goods. Games and Economic Behavior. forthcoming.

Carpenter, J., Burks, S., Verhoogen, E., 2005. Comparing students to workers: The effects of social framing on behavior in distribution games. In: Carpenter, J., Harrison, G., List, J. (Eds.), Field experiments in economics, JAI/Elsevier, Greenwich, Conn. and London, pp. 261-290.

Carpenter, J., Daniere, A., Takahashi, L., 2004a. Cooperation, trust, and social capital in southeast Asian urban slums. Journal of Economic Behavior \& Organization. 55, 533-551.

Carpenter, J., Daniere, A., Takahashi, L., 2004b. Social capital and trust in southeast Asian cities. Urban Studies. 41, 853-874.

Carter, M., Castillo, M., 2002. The economic impacts of altruism, trust and reciprocity: An experimental approach to social capital. AAE Staff Papers, University of Wisconsin-Madison. mimeo.

Charness, G., Rabin, M., 2002. Understanding social preferences with simple tests. Quarterly Journal of Economics. 117, 817-870.

Falk, A., Fehr, E., Fischbacher, U., 2003. On the nature of fair behavior. Economic Inquiry. 41, 20-26.

Fehr, E., Fischbacher, U., 2002. Why social preferences matter - the impact of non-selfish motives on competition, cooperation and incentives. Economic Journal. 112, 1-33.

Fehr, E., Gaechter, S., 2000a. Cooperation and punishment in public goods experiments. American Economic Review. 90, 980-994.

Fehr, E., Gaechter, S., 2000b. Fairness and retaliation: The economics of reciprocity. Journal of Economic Perspectives. 14, 159-181.

Fischbacher, U., Gaechter, S., Fehr, E., 2001. Are people conditionally cooperative? Evidence from a public goods experiment. Economic Letters. 71, 397-404.

Guion, R., Gibson, W., 1965. Validity of personality measures in personnel selection. Personnel Psychology. 18, 135-164. 
Harrison, G., List, J., 2004. Field experiments. Journal of Economic Literature. 42, 1009-1055.

Henrich, J., 2000. Does culture matter in economic behavior? Ultimatum game bargaining among the Machiguenga Indians of the Peruvian Amazon. American Economic Review. 90, 973-979.

Henrich, J., Boyd, R., Bowles, S., Camerer, C., Fehr, E., Gintis, H., McElreath, R., 2001. In search of homo economics: Behavioral experiments in 15 small-scale societies. American Economic Review. 91, 73-78.

Hollenbeck, J., Whitener, E., 1988. Reclaiming personality traits for personnel selection: Self-esteem as an illustrative case. Journal of Management. 14, 81-91.

Johnson, A., Luthans, F., Hennessey, H., 1984. The role of locus of control in leader influence behavior. Personnel Psychology. 37, 61-75.

Kandel, E., Lazear, E., 1992. Peer pressure and partnerships. Journal of Political Economy. 100, 801-817.

Karlan, D., 2004. Using experimental economics to measure social capital and predict financial decisions. American Economic Review. Forthcoming.

Ledyard, J., 1995. Public goods: A survey of experimental research. In: Kagel, J., Roth, A. (Eds.), The handbook of experimental economics, Princeton University Press, Princeton, pp. 111-194.

List, J., 2004. Young, selfish and male: Field evidence of social preferences. The Economic Journal. 114, 121-149.

Masclet, D., Noussair, C., Tucker, S., Villeval, M.-C., 2003. Monetary and nonmonetary punishment in the voluntary contributions mechanism. American Economic Review. 93, 366-380.

Platteau, J.-P., Seki, E., 1998. Coordination and pooling arrangements in Japanese coastal fisheries. Department of Economics, University of Namur, Working Paper No. 208.

Rotemberg, J.J., 1994. Human relations in the workplace. Journal of Political Economy. 102, 684-717.

Rotter, J.B., 1966. Generalized expectancies for internal versus external control of reinforcement. Psychological Monographs; General and Applied. 80, $1-28$.

Seki, E., 2004. Effects of rotation scheme on fishing behavior with price discrimination and limited durability: Theory and evidence. Department of Economics, University of Aberdeen Working Paper.

Szilagyi, A., Sims, H., Keller, R., 1976. Role dynamics, locus of control, and employee attitudes and behaviors. Academy of Management Journal. 19, $259-276$. 